\title{
Menopausa: fator de risco para doença periodontal?
}

\author{
Menopause: a risk factor for periodontal disease? \\ Débora Pallos ${ }^{1}$, Alessandro Ceschin², Gilberto de Abreu Victor ${ }^{3}$, Rogério Cardoso Bulhões ${ }^{4}$, \\ Maria Rozeli de Souza Quirino ${ }^{5}$
}

\section{RESUM0}

Objetivo: verificar se existe relação entre os parâmetros periodontais e os níveis de estrógeno e densitometria óssea mineral (DOM). Métodos: foram avaliadas 46 mulheres na pós-menopausa entre 44 e 68 anos de idade $(52,2 \pm 4,8)$ e 15 mulheres como grupo controle entre 35 e 54 anos de idade $(44,7 \pm 7,5)$. Parâmetros periodontais como profundidade à sondagem (PS), perda de inserção clínica (PIC) e dentes ausentes (DA) foram comparados com os níveis de estrógeno (suficiente e deficiente) e DOM em normais, osteopênicas e osteoporóticas. Os dados foram comparados pela diferença das médias entre os grupos e analisados pelo teste de Aspin-Welch. Resultados: as médias dos parâmetros de PS, PIC e DA, quando associados ao grau da DOM em normais $(2,1 \pm 0,5 ; 2,9 \pm 1,4$ e 10,6 $\pm 5,0)$, osteopênicas $(2,3 \pm 0,7 ; 3,0 \pm 1,1$ e 12,8 $\pm 5,1)$ e osteoporóticas $(2,4 \pm 0,6$; $2,7 \pm 0,9$ e 14,3 $\pm 5,7)$, não mostraram diferenças significativas ( $>>0,05)$. Foi encontrada diferença significante entre o grupo controle e nas mulheres menopausadas para PIC e DA. Quando comparados com os níveis de estrógeno os resultados demonstraram igualdade para os parâmetros periodontais. Conclusões: apesar de alguns estudos demonstrarem correlação positiva da doença periodontal com osteoporose e com os níveis de estrogênio, na população de mulheres menopausadas estes dados não foram confirmados neste estudo.

PALAVRAS-CHAVE: Periodontite; Menopausa; Densitometria; Estrógeno

\section{ABSTRACT}

Purpose: to verify if there is a relationship between the clinical periodontal parameters and estrogen levels and bone mineral density (BMD). Methods: forty-six post-menopausal women aged 44 to 68 years $(52.2 \pm 4.8)$ and 15 women aged 35 to 54 years $(44.7 \pm 7.5)$ were evaluated. Periodontal parameters like probing depth (PD), clinical attachment loss (CAL), and missing teeth (MT) were compared with estrogen levels (sufficient and insufficient) and BMD (normal, osteopenic and osteoporotic). Data of the mean difference between the groups were compared by the Aspin-Welch test. Results: the means of PD, CAL and MT, when associated with the normal $(2.1 \pm 0.5 ; 2.9 \pm 1.4$ and $10.6 \pm 5.0)$, osteopenic $(2.3 \pm 0.7 ; 3.0 \pm 1.1$ and 12.8 \pm 5.1$)$ and osteoporotic BMD $(2.4 \pm 0.6 ; 2.7 \pm 0.9$ and $14.3 \pm 5.7)$, did not show statistical difference (p>0.05). A significant difference was found between the control group and postmenopausal women for CAL and MT. When compared with the estrogen levels the results did not show a difference regarding the periodontal parameters. Conclusion: although some studies showed a positive correlation with osteoporosis and estrogen level, in this population of menopausal women these findings were not confirmed.

KEYWORDS: Periodontites; Menopause; Desintometry, x-ray; Estrogen

\section{Introdução}

Periodontite é definida como inflamação dos tecidos de suporte dos dentes de caráter destrutivo e progressivo, caracterizada pela reabsorção do osso alveolar e perda do tecido de inserção dental, e é con-

Apoio FAPESP - Projeto: 02/12891-2

1 Professora Assistente Doutor da Disciplina de Periodontia do Departamento de Odontologia da Universidade de Taubaté - UNITAU - Taubaté (SP), Brasil.

2 Professor do Curso de Especialização Escola de Aperfeiçoamento Profissional - Associação Paulista de Cirurgião Dentista Santo André (SP), Brasil.

3 Chefe do Setor de Ginecologia e Obstetrícia, Hospital Universitário, Departamento de Ginecologia - Universidade de Taubaté - UNITAU - Taubaté (SP), Brasil.

4 Cirurgião-dentista e Gerente de Compensação e Desenvolvimento de Pessoas e Consultor Free-Lance em Matemática, Estatística e Sistemas de Gestão

4 Cirurgião-dentista e Gerente de Compensação e Desenvolvimento de Pessoas e Consultor Free-Lance em Matemática, Estatística e Sistemas de Gestão Taubaté (SP), Brasil.

Correspondência: Débora Pallos

Rua Ásia 173 - São Paulo - 05413030 - São Paulo - SP - Fone (11) 3085-1800 - e-mail: dpallos@netpoint.com.br

Recebido em: 10/11/2005

Aceito com modificações em: 5/6/2006

Rev Bras Ginecol Obstet. 2006; 28(5): 292-7 
siderada a maior causa de perda dental e edentulismo em adultos. A etiologia da doença periodontal associada a infecção bacteriana está bem estabelecida, como agentes etiológicos podem ser citados microrganismos subgengivais como Porphiromonas gingivallis, Prevotella intermedia, Bacteroides forsythus, Actinobacillus actinomycetemcomitans e espiroquetas. A suscetibilidade do hospedeiro a esses agentes bacterianos também pode ter importante papel na sua prevalência e progressão ${ }^{1}$.

Fatores de risco associados à doença periodontal incluem aumento da idade, presença de biofilme dentário ou placa bacteriana patogênica, disfunção imunológica, deficiência nutricional, uso de medicação como o corticóide, gênero, estresse, tabaco, genética e condições sistêmicas incluindo alterações de neutrófilos, diabete, gravidez, alterações hormonais e osteoporose ${ }^{2}$.

A menopausa é caracterizada pela interrupção da menstruação que ocorre por volta dos 50 anos de idade. O período de transição entre o ciclo menstrual normal e a amenorréia completa varia de menos de um ano a onze anos, com a média permanecendo por volta de quatro anos. Uma das mais importantes características da menopausa é a significante diminuição da produção e secreção dos hormônios ovarianos, principalmente o estrógeno, que é considerado um dos fatores patogênicos dominantes para a osteoporose em mulheres. Ocorre também decréscimo na absorção de cálcio da dieta e um aumento dos níveis de cálcio na urina. A osteoporose, por sua vez, se caracteriza pela redução da massa óssea e pela deterioração da microarquitetura tanto do osso cortical como trabecular, resultante de desequilíbrio entre a reabsorção e a aposição óssea.

A perda óssea é um fator de risco comum à doença periodontal e tanto a doença periodontal como a osteoporose começam a mostrar seus efeitos após 35 anos de idade. Além disso, tem sido sugerido que fatores sistêmicos responsáveis pela osteoporose podem interagir com fatores locais (doença periodontal) aumentando o padrão de perda óssea alveolar ${ }^{3}$. Um fator que tem sido relacionado com esta interação é o efeito da alteração dos níveis de estrógeno relacionado com o aumento da reabsorção óssea alveolar ${ }^{4}$.

Por ser doença multifatorial, a osteoporose tem sido apontada como um dos fatores de risco para a doença periodontal, mas os estudos com população e de mulheres nestas condições têm sido controversos.

Portanto, a proposição deste trabalho foi avaliar as condições periodontais encontradas em mulheres na menopausa, comparando parâmetros clínicos periodontais com os níveis de estrógeno e a condição de osteoporose.

Métodos

Este estudo do tipo transversal foi conduzido na Universidade de Taubaté - UNITAU. As participantes foram selecionadas tanto na Clínica Odontológica do Departamento de Odontologia da UNITAU como na Clinica de Climatério do Hospital Universitário da UNITAU. Todas foram convidadas a participar deste estudo após aprovação do CEP/UNITAU n ${ }^{\circ}$ 319/02 e assinaram o termo de consentimento livre e esclarecido. Para inclusão no grupo, a mulher já deveria estar na menopausa e apresentar no mínimo 10 dentes presentes na cavidade bucal. Como critério de exclusão, não poderia participar quem apresentasse menopausa precoce ou doenças sistêmicas graves e câncer. No estudo poderiam participar mulheres com diferentes níveis de doença periodontal e de densitometria óssea mineral (DOM), portanto a gravidade destas não foi critério de seleção. Para o grupo controle, foram convidadas mulheres com mais de 35 anos de idade sem história de menopausa, com no mínimo 10 dentes presentes na cavidade bucal e com ausência de doenças sistêmicas graves.

No grupo de mulheres na menopausa, a avaliação médica foi realizada pelo médico ginecologista do Hospital Universitário da UNITAU, que as classificou pelo índice menopáusico de Blatt e Kupperman por meio dos sintomas apresentados; foi verificado o nível de $\mathrm{E}_{2}$ sérico (17- $\beta$ estradiol/ $\mathrm{mL}$ ), por meio de exame laboratorial de dosagem de 17- $\beta$ estradiol $/ \mathrm{mL}$, sendo consideradas estrógeno-deficientes as pacientes com nível de $\mathrm{E}_{2}$ inferior a $24 \mathrm{pg} / \mathrm{mL}$. A DOM foi avaliada na espinha lombar (L1-LA), na região da bacia, na coluna lombar e no colo do fêmur. Os valores foram expressos como um T-score (desvio padrão de valores observados de DOM do pico de massa óssea de mulher saudável de 30 anos de idade), definindose osteopenia como T-score entre -1.0 e -2.5 e osteoporose como T-score menor que -2.5.

Todas foram examinadas na Clínica Odontológica da Universidade de Taubaté e os dados obtidos na anamnese e no exame clínico foram registrados em ficha clínica previamente elaborada.

Foram verificados os seguintes parâmetros periodontais: profundidade à sondagem (PS) obtida por um único examinador em seis sítios por dente (mésio-vestibular, médio, disto-vestibular e 
mésio-lingual, médio e disto-lingual) usando a sonda periodontal manual do tipo Williams $\left(\right.$ Newmar $\left.^{\circledR}\right)$. A profundidade foi mensurada da margem gengival livre até a base da bolsa periodontal. A perda de inserção clínica (PIC) foi obtida de todos os sítios examinados por meio da medida da distância da junção esmalte-cemento (JEC) até a margem gengival (MG) somando a medida da profundidade à sondagem. Resumindo: PIC = PS + (JEC a MG). Para a comprovação da perda óssea alveolar, radiografias do tipo periapicais e panorâmicas foram feitas quando necessário. $\mathrm{O}$ examinador periodontal era do tipo simples cego, pois não conhecia nem a condição hormonal nem os valores da DOM das participantes.

Após o exame clínico periodontal, foram diagnosticadas e classificadas de acordo com o seu nível de perda de inserção clínica em periodontite crônica leve (PIC de 1 a $2 \mathrm{~mm}$ ), moderada (PIC de 3 a $4 \mathrm{~mm}$ ) e avançada (PIC $\geq 5 \mathrm{~mm}$ ), seguindo a última classificação proposta pela Academia Americana de Periodontia ${ }^{1}$.

Quarenta e seis mulheres na menopausa foram incluídas no estudo com idade variando de 44 a 68 anos (média de idade $52,2 \pm 4,8$ ). Foram incluídas no grupo controle 15 mulheres não menopausadas com idade de 35 a 54 anos (média de idade $44,7 \pm 7,5$ ).

Com os resultados obtidos do nível de estrógeno as mulheres menopausadas foram então divididas em suficiente $(n=15)$ e deficiente $(n=31)$ e outra divisão foi feita com base no resultado da DOM em normal $(n=19)$, osteopênicas $(n=23)$ e com osteoporose $(n=4)$.

Os dados foram comparados com relação ao grau de destruição periodontal encontrada nas pacientes. As diferenças das médias encontradas entre os grupos foram analisadas pelo teste de hipóteses de Aspin-Welch com um nivel de confiança de $95 \%$.

\section{Resultados}

Primeiramente foram comparados os grupos de acordo com os níveis de estrógeno e grupo controle. Houve diferença estatisticamente significativa para os parâmetros de perda de inserção e dentes ausentes (DA) na comparação do grupo controle com os grupos estrógeno suficiente e deficiente. No grupo controle foi encontrada média de ausência dental de 7,6 dentes, no grupo do nível de estrógeno suficiente de 12,5 dentes e no grupo estrógeno deficiente de 11,8 dentes. Já quanto à perda de inserção encontrou-se média de 1,7 mm de perda para o grupo controle, e para os grupos estrógeno suficiente e deficiente, de 3,0 e $2,8 \mathrm{~mm}$, respectivamente. Pôde-se notar que o parâmetro de profundidade de sondagem não apresentou diferença significativa em nenhum grupo avaliado (Tabela 1).

Tabela 1 - Valores médios e desvios padrões de idade, dentes ausentes (DA), profundidade a sondagem (OS), mm) e perda de inserção clínica (PIC, mm) das participantes agrupadas quanto ao nível de estrógeno.

\begin{tabular}{lccc}
\hline & Controle & \multicolumn{2}{c}{ Menopausa } \\
& & Suficiente & Deficiente \\
\hline Idade & $44,7 \pm 7,5$ & $52,4 \pm 4,8$ & $52,1 \pm 4,9$ \\
DA & $7,6 \pm 5,5^{\mathrm{a}}$ & $12,5 \pm 5,3^{\mathrm{b}}$ & $11,8 \pm 5,2^{\mathrm{b}}$ \\
PS & $2,6 \pm 0,9^{\mathrm{a}}$ & $2,1 \pm 0,6^{\mathrm{a}}$ & $2,3 \pm 0,6^{\mathrm{a}}$ \\
PIC & $1,7 \pm 1,5^{\mathrm{a}}$ & $3,0 \pm 1,4^{\mathrm{b}}$ & $2,8 \pm 1,1^{\mathrm{b}}$ \\
\hline
\end{tabular}

Valores seguidos por letras minúsculas distintas (sentido horizontal) diferem estatisticamente entre si $(p<0,05)$.

Quando comparados os grupos de acordo com o resultados da DOM (normal, osteopenia, osteoporose) e o grupo controle, somente houve diferença significativa entre as médias dos grupos osteopenia com o grupo controle quanto aos parâmetros PIC $(1,7 \mathrm{~mm}$ e $3,0 \mathrm{~mm})$ e DA (7,6 e 12,8 dentes) e entre as médias de PIC para o grupo normal $(1,7 \mathrm{~mm})$ comparado com o grupo controle $(2,8 \mathrm{~mm})(\mathrm{p}<0,05)$. Os outros parâmetros avaliados não apresentaram diferenças significativas (Tabela 2).

Tabela 2 - Valores médios e desvios padrões de idade, dentes ausentes (DA), profundidade a sondagem (OS, mm) e perda de inserção clínica (PIC, mm) das participantes agrupadas quanto ao resultado da densitometria óssea mineral.

\begin{tabular}{lcccc}
\hline & Controle & \multicolumn{3}{c}{ Menopausa } \\
& & \multicolumn{3}{c}{ Densitometria óssea mineral } \\
& & Normal & Osteopenia & Osteoporose \\
\hline & $44,7 \pm 7,5^{\mathrm{a}}$ & $51,4 \pm 3,9^{\mathrm{a}}$ & $52,2 \pm 4,5^{\mathrm{a}}$ & $56,0 \pm 9,2^{\mathrm{a}}$ \\
DA & $7,6 \pm 5,5^{\mathrm{a}}$ & $10,6 \pm 5,0^{\mathrm{a}}$ & $12,8 \pm 5,1^{\mathrm{b}}$ & $14,2 \pm 5,7^{\mathrm{a}}$ \\
PS & $2,6 \pm 0,9^{\mathrm{a}}$ & $2,2 \pm 0,5^{\mathrm{a}}$ & $2,3 \pm 0,7^{\mathrm{a}}$ & $2,4 \pm 0,6^{\mathrm{a}}$ \\
PIC & $1,7 \pm 1,5^{\mathrm{a}}$ & $2,8 \pm 1,4^{\mathrm{b}}$ & $3,0 \pm 1,1^{\mathrm{b}}$ & $2,7 \pm 0,9^{\mathrm{ab}}$ \\
\hline
\end{tabular}

Valores seguidos por letras minúsculas distintas (sentido horizontal) diferem estatisticamente entre si $(p<0,05)$.

No grupo das mulheres menopausadas somente quatro apresentavam osteoporose, portanto nova análise foi feita para verificar se existia diferença entre o grupo de menopausa com osteopenia/ osteoporose comparado com normal e com o grupo controle. Somente foi encontrada diferença significativa entre normal e controle quanto ao parâmetro PIC (1,7 e 2,8 mm, respectivamente) e entre os grupos osteopenia/osteoporose e controle quanto aos parâmetros PIC (1,7 e 2,9 mm) e DA (7,6 e 13,0 dentes ausentes) $(\mathrm{p}<0,05)($ Tabela 3$)$. 
Tabela 3 - Valores médios e desvios padrões de idade, dentes ausentes (DA) profundidade a sondagem (OS, mm) e perda de inserção clínica (PIC, mm) das participantes agrupadas quanto ao resultado da densitometria óssea mineral, agrupando-se osteopenia e osteoporose.

\begin{tabular}{cccc}
\hline & Controle & \multicolumn{2}{c}{$\begin{array}{c}\text { Menopausa } \\
\text { Densitometria óssea mineral } \\
\end{array}$} \\
& & Normal & Osteopenia/Osteoporose \\
\hline & $44,7 \pm 7,5^{\mathrm{a}}$ & $51,4 \pm 3,9^{\mathrm{a}}$ & $52,7 \pm 5,4^{\mathrm{a}}$ \\
DA & $7,6 \pm 5,5^{\mathrm{a}}$ & $10,6 \pm 5,0^{\mathrm{a}}$ & $13,0 \pm 5,1^{\mathrm{b}}$ \\
PS & $2,6 \pm 0,9^{\mathrm{a}}$ & $2,2 \pm 0,5^{\mathrm{a}}$ & $2,3 \pm 0,7^{\mathrm{a}}$ \\
PIC & $1,7 \pm 1,5^{\mathrm{a}}$ & $2,8 \pm 1,4^{\mathrm{b}}$ & $2,9 \pm 1,1^{\mathrm{b}}$
\end{tabular}

Valores seguidos por letras minúsculas distintas (sentido horizontal) diferem estatisticamente entre si $(p<0,05)$.

\section{Discussão}

A menopausa é caracterizada pela interrupção da menstruação que acontece por volta dos 50 anos de idade e, embora seja reconhecida há séculos, só recentemente tem recebido maior atenção não apenas pelos seus sintomas, mas também pelo fato de que a diminuição da secreção dos hormônios ovarianos, principalmente o estrógeno, constitui fator preponderante da patogênese da osteoporose $\mathrm{e}^{4,5}$.

A osteoporose na pós-menopausa, juntamente com a fase senil do indivíduo, é forma primária de osteoporose e considerada evolucional, constituindo a mais comum das doenças ósseas metabólicas que afetam muitas mulheres no final de suas vidas. No entanto, não podemos deixar de lado as causas secundárias, como as afecções de origem endócrino-metabólicas (hereditárias ou não), reumatológicas, renais, digestivas, neoplásicas e o uso de medicações que interferem com o equilíbrio do cálcio tais como corticosteróides, anticonvulsivantes, antiácidos, entre outros. Portanto, sendo a osteoporose doença sistêmica, pode afetar também os ossos maxilares ${ }^{3,6,7}$.

A doença periodontal constitui uma inflamação crônica dos tecidos de suporte dos dentes, iniciada por uma microflora gram-negativa, resultando em destruição do tecido conjuntivo e osso. A presença de lipopolissacarídeos e endotoxinas derivadas dos microrganismos (gram-negativas) ativam várias células do hospedeiro a liberar citocinas inflamatórias como a interleucina 1 (IL1). A IL-1 $1 \beta$ é um dos mais potentes mediadores de reabsorção óssea, estimulando a diferenciação e ativação dos osteoclastos e inibindo também a formação óssea ${ }^{8,9}$.

Sabe-se que os estrogênios podem inibir os mediadores e mecanismos celulares da inflamação, o que leva a crer que a deficiência desse hormônio pode alterar a resposta aos produtos da placa bacteriana ${ }^{4,6,7}$. Na cavidade bucal os principais sintomas relacionados à menopausa são sensação de desconforto, incluindo dor, ardência, alterações de paladar, secura da boca e perda óssea alveolar resultante da osteoporose $\mathrm{e}^{10}$.

A relação entre doença periodontal e osteopenia/osteoporose ainda não está bem estabelecida. Vários estudos foram desenvolvidos para determinar esta associação. Alguns mostram que a osteoporose não é fator etiológico da periodontite, mas pode afetar a gravidade da doença periodontal preexistente ${ }^{11}$. Outros não observaram significante correlação entre parâmetros clínicos de periodontite e a medida de massa óssea esquelética, sugerindo então que esta relação não era tão importante. No entanto, não excluíram que a manifestação da periodontite avançada poderia estar relacionada com osteoporose ${ }^{12}$. A observação radiográfica demonstrou que a osteoporose tem efeito sobre o osso alveolar com decréscimo de massa óssea, demonstrando que a perda óssea generalizada devido à osteoporose torna a maxila suscetível à aceleração na reabsorção do osso alveolar ${ }^{4,13}$. Pode-se notar que a literatura é controversa com respeito à associação da doença periodontal com osteoporose, mostrando ser campo extenso para estudos comparativos em diversas populações ${ }^{14}$.

Ao correlacionarmos as médias de idades e perda de inserção em mulheres na menopausa, a literatura mostra variabilidade das mesmas, como podemos observar em estudo em que as mulheres apresentavam média de idade de 58 anos e 2,0 mm de perda de inserção ${ }^{15}$ e em outro que encontrou média de 62 anos de idade e $2,7 \mathrm{~mm}$ de perda de inserção ${ }^{4}$. Ao compararmos estes achados com os do presente estudo com média de idade de 52 anos e 3,0 mm de perda de inserção, verificamos que este apresenta menor média de idade, mas com perda de inserção maior, demonstrando nesta população a presença de doença periodontal mais avançada. Assim, essa diferença encontrada poderia ser justificada considerando que as participantes desta pesquisa são de baixa renda e com maior dificuldade de ter acesso ao tratamento odontológico.

Por meio da avaliação periodontal, foi verificada também a freqüência de dentes ausen-

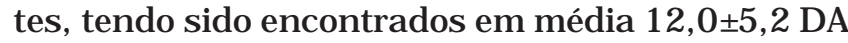
por paciente na menopausa, sendo os primeiros molares superiores e inferiores e o segundo molar inferior direito os dentes ausentes mais prevalentes; já o grupo controle apresentou média de $7,6 \pm 5,5$, demonstrando menor perda dos elementos dentais. 
Outros estudos demonstraram valores diferentes nas médias de dentes remanescentes, como $24,0 \pm 4,0^{15}$ e $23,6 \pm 4,5^{4}$, e maior freqüência de ausência dos primeiros molares inferiores. Estes dados também demonstram grande diferença das populações estudadas quanto aos cuidados de saúde bucal.

Outro fator de risco para a perda dentária é a osteoporose. Estudos mostraram que a DOM está fortemente correlacionada com o número de dentes remanescentes em mulheres na pós-menopausa e sugerem que a perda óssea sistêmica contribui para a perda dos dentes ${ }^{16-19}$. Porém, um estudo seccional não encontrou diferença significativa quando utilizados os parâmetros clínicos periodontais em 292 mulheres osteopênicas ${ }^{20}$ no qual foram encontrados média de dentes presentes de $17,2 \pm 7$ e PIC no valor de $2,8 \pm 0,9 \mathrm{~mm}$. O presente estudo demonstrou uma semelhança na média de dentes ausentes $(12,8 \pm 5,1)$ e de perda de inserção $(2,9 \pm 1,1)$.

Comparando a perda de inserção clínica e o número de dentes ausentes no grupo controle e de mulheres menopausadas, verificamos diferenças significativas, mostrando melhores condições no grupo controle, fatos estes que vem ao encontro de alguns estudos que demonstram a influência da alteração hormonal na atividade óssea, indicando que a periodontite e a perda dental na menopausa possam ser indicadores de osteopenia/osteoporose. No entanto, analisando os grupos de mulheres estrógeno suficientes e deficientes, os parâmetros periodontais não mostraram tal associação, como apresentado em estudos semelhantes ${ }^{13,16,21}$. Estes estudos concluíram que a osteopenia na pós-menopausa constitui fator de risco para a doença periodontal. Porém, em estudo preliminar conduzido por este grupo ${ }^{22}$, com mulheres estrógeno deficientes, não foi encontrada esta associação.

Uma das dificuldades encontradas na seleção das participantes deste estudo foi a condição de apresentarem um mínimo de 10 dentes na cavidade bucal. A maioria das mulheres que procuram o ambulatório de Climatério do Hospital Universitário da UNITAU eram desdentadas. Este fato reflete a realidade e as condições socioculturais dos indivíduos que procuram atendimento em ambulatórios e clínicas públicas.

Quanto aos resultados encontrados relacionando doença periodontal, perda dental e valores de DOM, estudo longitudinal que acompanhou por dois anos 398 mulheres ${ }^{23}$ não encontrou diferença significativa. Entretanto, em outro estudo, quando avaliados os valores da DOM da região de cálcis, associação positiva foi encontrada na comparação com a ausência dental ${ }^{24}$.
Portanto, sendo a doença periodontal comum na população e sabidamente relacionada a doenças sistêmicas, uma via de mão dupla, fazem-se necessários estudos cada vez mais profundos para a melhor compreensão de sua patogênese e conseqüentemente do desenvolvimento de novos caminhos para o controle da doença.

\section{Agradecimentos}

Ao laboratório de Análises Clínicas Osvaldo Cruz e Clínica de Fraturas Santa Terezinha. Apoio: Fundação de Amparo à Pesquisa do Estado de São Paulo e Fundo de Apoio ao Ensino e Pesquisa - Faculdade de Ciências Médicas - Universidade Estadual de Campinas (FAEP 008/98).

\section{Referências}

1. Armitage GC. Development of classification system for periodontal disease and conditions. Ann Periodontol. 1999;4(1):1-6.

2. Wactawski-Wende J, Grossi SG, Trevisan M, Genco RJ, Tezal M, Dunford RG, et al. The role of osteopenia in oral bone loss and periodontal disease. J Periodontol. 1996;67(10 Suppl):1076-84.

3. Hildebolt CF, Pilgram TK, Yokoyama-Crothers N, Vannier MW, Dotson M, Muckerman J, et al. Alveolar bone height and postcranial bone mineral density: negative effects of cigarette smoking and parity. J Periodontol. 2000;71(5):683-9.

4. Tezal M, Wactawski-Wende J, Grossi SG, Ho AW, Dunford R, Genco RJ. The relationship between bone mineral density and periodontitis in postmenopausal women. J Periodontol. 2000;71(9):1492-8.

5. Albuquerque CFM, Pinto-Neto AM, Lowczyk DJ, Pallos D. Doença periodontal e osteoporose em mulheres na pós-menopausa. Femina. 2004;32(4):315-21.

6. Lopez-Marcos JF, Garcia-Valle S, Garcia-Iglesias AA. Periodontal aspects in menopausal women undergoing hormone replacement therapy. Med Oral Patol Oral Cir Bucal. 2005;10(2):132-41.

7. Taguchi A, Sanada M, Suei Y, Ohtsuka M, Nakamoto $\mathrm{T}$, Lee $\mathrm{K}$, et al. Effect of estrogen use on tooth retention, oral bone height, and oral bone porosity in Japanese postmenopausal women. Menopause. 2004;11(5):556-62.

8. Engebretson SP, Grbic JT, Singer R, Lamster IB. GCF IL-1b profiles in periodontal disease. J Clin Periodontol. 2002;29(1):48-53. 
9. Tsai CC, Ho YP, Chen CC. Levels of interleukin-1b and interleukin-8 in gingival crevicular fluids in adult periodontitis. J Periodontol. 1995;66(10):8529.

10. Payne JB, Zachs NR, Reinhardt RA, Nummikoski $\mathrm{PV}$, Patil K. The association between estrogen status and alveolar bone density changes in postmenopausal women with a history of periodontitis. J Periodontol. 1997;68(1):24-31.

11.Steinberg BJ. Women's oral health tissues. J Dent Educ. 1999;63(3):271-5.

12. Elders PJ, Habets LL, Netelenbos JC, van der Linden LW, van der Stelt PF. The relation between periodontitis and systemic bone mass in women between 46 and 55 years of age. J Clin Periodontol. $1992 ; 19(7): 492-6$

13. Inagaki K, Kurosu Y, Yoshinari N, Noguchi T, Krall EA, Garcia RI. Efficacy of periodontal disease and tooth loss to screen for low bone mineral density in Japanese women. Calcif Tissue Int. 2005;77(1):914.

14. Reddy MS. Oral osteoporosis: is there an association between periodontitis and osteoporosis? Compend Contin Educ Dent. 2002;23(10 Suppl):21-8.

15. Hildebolt CF, Pilgram TK, Dotson M, YokoyamaCrothers N, Mucherman J, Hauser J, et al. Attachment loss with postmenopausal age and smoking. J Periodontal Res. 1997;32(7):619-25.

16. Hildebolt CF, Pilgram TK, Dotson M, ArmamentoVillareal R, Hauser J, Cohen S, et al. Estrogen and/ or calcium plus vitamin $\mathrm{D}$ increase mandibular bone mass. J Periodontol. 2004;75(6):811-6.
17. Bando K, Nitta H, Matsubara M, Ishikawa I. Bone mineral density in periodontally healthy and edentulous postmenopausal women. Ann Periodontol. 1998;3(1):322-6.

18. Mariotti AJ, Minenna L. Brittle bones and loose teeth: relationship between osteoporosis and periodontitis. Alpha Omegan. 2003;96(4):41-6.

19. Krall EA, Dawson-Hughes B, Papas A, Garcia RI. Tooth loss and skeletal bone density in healthy postmenopausal women. Osteoporos Int. 1994;4(2):104-9.

20.Weyant RJ, Pearlstein ME, Churak AP, Forrest K, Famili P, Cauley JA. The association between osteopenia and periodontal attachment loss in older women. J Periodontol. 1999;70(9):982-91.

21. Reinhardt RA, Payne JB, Maze CA, Patil KD, Gallagher SJ, Mattson JS. Influence of estrogen and osteopenia/osteoporosis on clinical periodontitis in postmenopausal women. J Periodontol. 1999;70(8):823-8.

22. Ceschin A, Pallos D, Victor GA, Jardim JCM, Quirino MRS. Avaliação dos niveis de interleucina $1 \mathrm{~b} \mathrm{em}$ mulheres na menopausa com doença periodontal. Rev Assoc Paul Cir Dent. 2005;59(1):29-34.

23. Famili P, Cauley J, Suzuki JB, Weyant R. Longitudinal study of periodontal disease and edentulism with rates of bone loss in older women. J Periodontol. 2005;76(1):11-5.

24. Mohammad AR, Hoper DA, Vermilyea SG, Mariotti A. An investigation of the relationship between systemic bone density and clinical periodontal status in post-menopausal Asian-American women. Int Dent J. 2003;53(3):121-5. 